MATERIALS

\section{Rolling out data storage}

The data-storage capacity of CDs and DVDs is constrained by their size, so a group of researchers has devised a continuous process that makes long sheets of a many-layered optical storage medium.

Kenneth Singer of Case Western Reserve University in Cleveland, Ohio, and his colleagues began by melting and stacking two polymer layers and then pushing them through a series of 'multipliers', each of which doubles the number of layers. After the multi-layer melt was spread into a thin but dense optical-storage film, it could be cut and formed into various shapes and sizes. The team then used a laser to write data on each of 23 layers.

The group hopes that the method will eventually produce low-cost optical data film hundreds of metres long that can store terabytes or even petabytes of data roughly equivalent to one million DVDs.

Adv. Mater. http://dx.doi. org/10.1002/adma.201200669 (2012)

\section{CANCER IMMUNOLOGY}

\section{Pathway from breast to bone}

The likelihood that breast tumours will spread to the bone - a potentially deadly process - is enhanced when an immune-signalling pathway in the cancer cells is blocked.

Belinda Parker at the Peter MacCallum Cancer Centre in Melbourne, Paul Hertzog at Monash University in Clayton, both in Australia, and their team compared gene expression in cells

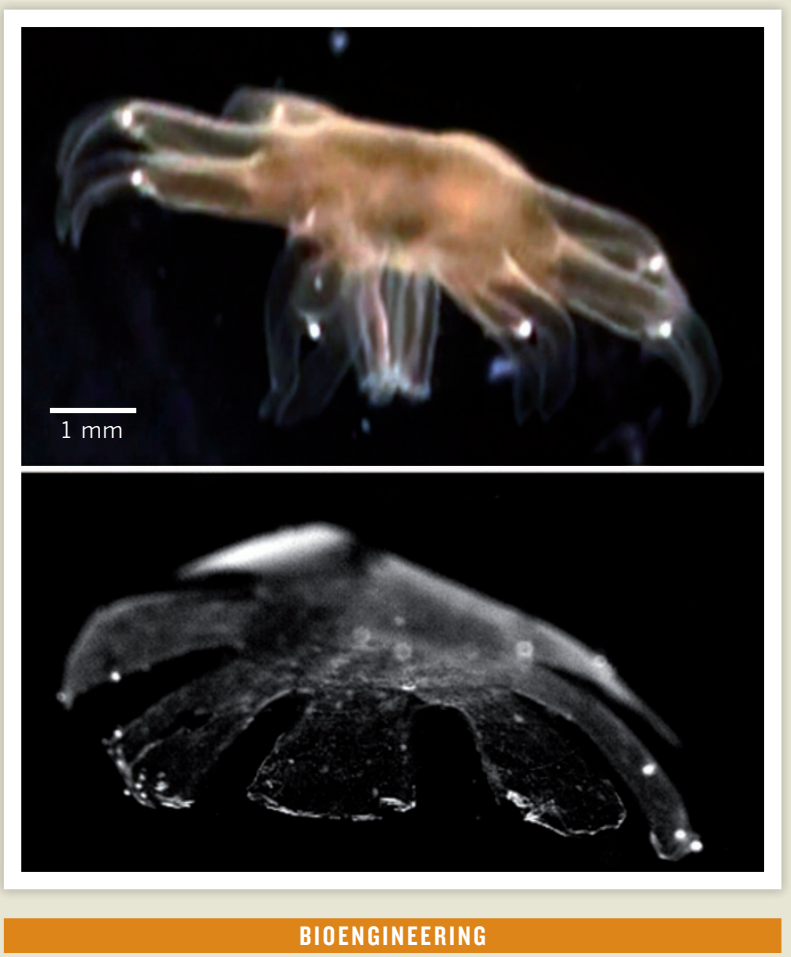

\title{
Engineered 'jellyfish'
}

By combining rat tissue and silicone, researchers have engineered a device that mimics the movements of jellyfish.

Kevin Kit Parker at Harvard University in Cambridge, Massachusetts, John Dabiri at the California Institute of Technology in Pasadena and their colleagues used computer imaging and tissue experiments to characterize the muscular structure of jellyfish (pictured top) and the motion - a quick contraction and slow recoil - that the animals use to feed and to propel themselves through water. By growing rat heart-muscle cells on a flexible silicone frame, the team then built a pump (bottom) capable of mimicking jellyfish shape, movement and fluid dynamics. Applying an electrical field to a water bath containing the 'jellyfish' caused it to contract with a power and at a rate similar to those of natural jellyfish.

The researchers suggest that the same design strategy could be used to produce other synthetic muscular pumps or models of simple organisms.

Nature Biotechnol. http://dx.doi.org/10.1038/nbt.2269 (2012)

For a longer story on this research, see go.nature.com/xfr6jc

from primary mouse breast tumours with those from bone metastases. They found that in the metastases, many genes sharing the same pathways as a protein called
IRF7 were suppressed. This protein regulates the body's responses to an immunesignalling molecule called interferon, a version of which is used in the treatment of certain cancers. Restoring IRF7 signalling in tumour cells reduced bone metastases and boosted immune activity and metastasis-free survival time.

Patients with breast cancer and low expression levels of IRF7-associated genes in their primary tumours could be at higher risk of metastasis, the authors say.

Nature Med. http://dx.doi. org/10.1038/nm.2830 (2012) MICROBIOLOGY

\section{Recycling at root} of arsenic 'life'

The reported discovery of a bacterial strain able to use arsenic in place of phosphorus to make essential molecules such as DNA generated much controversy and has recently been refuted. Murray Deutscher and his colleagues at the University of Miami in Florida provide an alternative explanation for the puzzling observation that bacteria of the strain Halomonadaceae GFAJ-1 grow when supplied with arsenate instead of phosphate.

The researchers used a radioactive isotope to label ribosomes - the cell's proteinmaking machinery - in the bacterium Escherichia coli. They found that arsenate causes ribosomes to degrade, a process that releases phosphate. After being cultured for about 80 hours in media that contained arsenate but not phosphate, a few arsenate-tolerant E. coli cells began to grow, slowly. Rather than growing by replacing phosphorus with arsenic, these cells recycled phosphorus released by deteriorating ribosomes, the authors suggest.

J. Biol. Chem. http://dx.doi. org/10.1074/jbc.C112.394403 (2012) 